\title{
Downregulation of RIP140 in triple-negative breast cancer inhibits the growth and proliferation of cancer cells
}

\author{
XIAO-HONG YU ${ }^{1}, \mathrm{XIAODONG} \mathrm{XUE}^{1}, \mathrm{XUN} \mathrm{ZHU}^{2}$ and $\mathrm{XIA} \mathrm{LI}{ }^{1}$ \\ ${ }^{1}$ Department of Breast Surgery, 903 Hospital, Jiangyou, Sichuan 421700; ${ }^{2}$ Department of General Surgery, \\ The Second Affiliated Hospital of Soochow University, Suzhou, Jiangsu 215006, P.R. China
}

Received May 4, 2017; Accepted December 21, 2017

DOI: $10.3892 / \mathrm{ol} .2018 .8434$

\begin{abstract}
The aim of the present study was to investigate the expression of receptor interacting protein 140 (RIP140) in triple-negative breast cancer (TNBC), and its association with the prognosis of patients with TNBC. A total of 179 patients with breast cancer were included in this study, with 41 cases of TNBC and 138 cases of non-TNBC. Immunohistochemical staining and western blotting were used to detect the protein expression of RIP140 in the cancerous and paracancerous tissues, revealing that expression of RIP140 was increased in TNBC tissues compared with non-TNBC tissues. High expression of RIP140 in breast cancer tissue was associated with a poorer survival time than low RIP140 expression. Using lentiviral transfection to downregulate RIP140 expression in MDA-MB-231 cells, the effects of RIP140 on the growth and proliferation of breast cancer cells was analyzed using subcutaneous tumors in BALB/c nude mice. Immunohistochemical staining using a Ki-67 antibody in subcutaneous tumor tissue was used to assess the proliferation of MDA-MB-231 cells. The short hairpin RNA-mediated downregulation of RIP140 in MDA-MB-231 cells suppressed the growth and the proliferation of subcutaneous tumors in BALB/c nude mice. Downregulation of RIP140 in breast cancer cells may therefore inhibit the growth and the proliferation of these cells, and may provide a therapeutic target for TNBC.
\end{abstract}

\section{Introduction}

Breast cancer is one of the most commonly diagnosed malignant tumors in women, and, based on data from 2011,

Correspondence to: Dr Xun Zhu, Department of General Surgery, The Second Affiliated Hospital of Soochow University, 1055 Sanxiang Road, Gusu, Suzhou, Jiangsu 215006, P.R. China E-mail: zhuxun1023@126.com

Dr Xia Li, Department of Breast Surgery, 903 Hospital, 113 Middle Position of Taiping Road, Jiangyou, Sichuan 421700, P.R. China E-mail: 823973570@qq.com

Key words: triple-negative breast cancer, receptor interacting protein 140 , proliferation, prognosis its incidence is increasing (1). Triple negative breast cancer (TNBC) is characterized by the negative expression of the estrogen receptor, progesterone receptor and human epidermal growth factor receptor 2, and accounts for $10-20 \%$ breast cancer cases worldwide $(1,2)$. Characteristically, TNBC is associated with early age of onset, high degree of malignancy, high recurrence rate, early metastasis and poor prognosis $(2,3)$. Receptor interacting protein 140 (RIP140) is a nuclear receptor transcriptional co-regulator that controls the transcription of target genes in several tissues, including adipose, skeletal muscle, cardiac muscle, liver and tumor tissues $(4,5)$. It has been demonstrated to serve notable roles in metabolism, inflammation and tumor development. RIP140 is a metabolic switch that regulates a number of metabolic pathways. As a co-repressor, RIP140 facilitates high-fat diet-induced obesity and induces insulin resistance. As a co-activator, RIP140 activates nuclear factor (NF)- $\mathrm{KB}$ and promotes the expression of pro-inflammatory cytokines, including tumor necrosis factor (TNF)- $\alpha$ and interleukin (IL)-6 in immune cells (4-6). RIP140 also affects tumorigenesis and tumor metastasis via E2F transcription factor and Wnt/adenomatous polyposis coli (APC)/ $\beta$-catenin signaling pathways $(5,7)$. Aziz et al (8) demonstrated that inhibition of RIP140 inhibited the growth of breast cancer cells in vivo and in vitro. However, to the best of our knowledge, no previous research has investigated the association between the expression of RIP140 in TNBC and the postoperative prognosis of patients with TNBC. Thus, the aim of the present study was to investigate the expression of RIP140 in TNBC, and the association between RIP140 expression and postoperative prognosis of patients with the disease.

\section{Materials and methods}

Cell culture and lentivirus transfection. The TNBC cell line, MDA-MB-231, was purchased from the Cell Bank of Type Culture Collection of the Chinese Academy of Sciences (Shanghai, China) and maintained in Dulbecco's Modified Eagle's Medium (DMEM; HyClone; GE Healthcare, Chicago, IL, USA) supplemented with $10 \%$ fetal bovine serum (FBS; HyClone; GE Healthcare Life Sciences, Logan, UT, USA) and $1 \%$ penicillin and streptomycin (HyClone; GE Healthcare Life Sciences) at $37^{\circ} \mathrm{C}$ in $5 \% \mathrm{CO}_{2}$.

The short hairpin RNA (shRNA)-RIP140 lentivirus and empty lentivirus were purchased from Shanghai GeneChem 
Co., Ltd. (Shanghai, China). Cells transfected with an empty lentivirus served as the negative control (NC) group, and untreated cells served as the control group. Lentiviral cell transduction was performed as previously described by Ho et al (6).

Clinical breast cancer samples. Tissue samples were collected from 179 patients with breast cancer who had undergone modified radical mastectomies at 903 Hospital (Jiangyou, China) between February 2005 and February 2008. Patient age ranged between 23 and 81 years old (median age, $54.2 \pm 19.7$ years). Postoperative pathological examination defined 41 cases of TNBC and 138 cases of non-TNBC. All tissues were used for immunohistochemistry and western blot analysis, with 10 samples of TNBC paracancerous tissue and 10 samples of non-TNBC paracancerous tissue from these patients also analyzed as controls. The clinicopathological information of the two groups of patients is provided in Table I. The present retrospective study was approved by the Research Ethics Committee of 903 Hospital (Jiangyou, China).

Western blot analysis. After $72 \mathrm{~h}$ of transfection of MDA-MB-231 cells, total protein was extracted using the Whole Protein Extraction kit (Nanjing KeyGen Biotech Co., Ltd., Nanjing, China), according to the manufacturer's protocol. The same method was used to extract protein from paracancerous, non-TNBC and TNBC tissues. The protein concentrations were determined using the BCA kit (Boster Biological Technology, Pleasanton, CA, USA), according to the manufacturer's protocols. Total cellular protein $(80 \mu \mathrm{g})$ was mixed with loading buffer (Boster Biological Technology) and boiled in $100^{\circ} \mathrm{C}$ for $5 \mathrm{~min}$, prior to protein separation by $8 \%$ SDS-PAGE. Proteins were then transferred onto polyvinylidene difluoride membranes (Merck KGaA, Darmstadt, Germany). Subsequent to blocking in $5 \%$ skim-milk, the membranes were incubated overnight at $4^{\circ} \mathrm{C}$ with anti-RIP140 (dilution, 1:1,000; catalog no. ab42126; Abcam, Cambridge, UK) or anti-GAPDH (dilution, 1:1,000; catalog no. sc293335; Santa Cruz Biotechnology, Inc., Dallas, TX, USA) primary antibodies in Tris-buffered saline with $0.01 \%$ Tween-20 (TBST) with 5\% bovine serum albumin (BSA). The following day, the membranes were incubated with the corresponding horseradish peroxidase (HRP)-conjugated mouse anti-goat secondary antibody (dilution, 1:2,000; catalog no. sc-516246; Santa-Cruz Biotechnology, Inc.) at $37^{\circ} \mathrm{C}$ for $30 \mathrm{~min}$ prior to washing three times with TBST. Protein signals were analyzed using Quantity One Software (version 4.62; Bio-Rad Laboratories, Inc., Hercules, CA, USA) following incubation with an enhanced chemiluminescence reagent (EMD Millipore, Billerica, MA, USA), according to the manufacturer's protocol. The PVDF membrane was purchased from Thermo Fisher Scientific, Inc. (Waltham, MA, USA).

Establishment of a subcutaneous breast cancer model in $B A L B / c$ nude mice. A total of 304 -week-old BALB/c nude mice $(15 \mathrm{~g})$ were purchased from Chongqing Medical University Experimental Animal Center (0005818; Chongqing, China). Mice were housed in a temperature at
Table I. RIP140 expression exhibited by TNBC and non-TNBC breast cancer tissues and basic clinicopathological patient information.

\begin{tabular}{lcccc}
\hline & \multicolumn{4}{c}{$\begin{array}{c}\text { RIP140 } \\
\text { expression }\end{array}$} \\
\cline { 3 - 4 } & & Patients, & Weak, & Strong, \\
Parameter & $\mathrm{n}$ & $\mathrm{n}$ & $\mathrm{n}$ & P-value \\
\hline Total & 179 & 115 & 64 & \\
TNBC & 41 & 6 & 35 & $<0.001$ \\
Non-TNBC & 138 & 109 & 29 & \\
Age, years & & & & 0.995 \\
$\geq 50$ & 141 & 78 & 63 & \\
$<50$ & 38 & 21 & 17 & \\
$\begin{array}{l}\text { Tumor } \\
\text { diameter, cm }\end{array}$ & & & & \\
$\geq 5$ & 61 & 28 & 33 & 0.069 \\
$<5$ & 118 & 71 & 47 & \\
Lymphatic & & & & \\
metastasis & & & & \\
$\quad$ Yes & 82 & 36 & 46 & 0.196 \\
No & 97 & 52 & 45 & \\
\hline
\end{tabular}

TNBC, triple-negative breast cancer; RIP140, receptor interacting protein 140.

$27^{\circ} \mathrm{C}, 50 \%$ relative humidity, alternately exposed to light for $10 \mathrm{~h}$ and without light for $14 \mathrm{~h}$ pathogen-free environment. All mice had free access to food and water. Subcutaneous tumors were generated by subcutaneous injection of $5 \times 10^{6}$ MDA-MB-231 cells (shRNA-RIP140, NC or control) under the right forelimb of each mouse. A total of 10 nude mice were sacrificed each week the tumors were excised for volume measurement and paraffin sectioning for nuclear proliferating antigen (Ki-67) immunohistochemistry. All experimental procedures were conducted in accordance with the Guide for the Care and Use of Laboratory Animals and approved by the ethical guidelines for animal experiments of 903 Hospital.

Immunohistochemistry. The expression of RIP140 in clinical breast cancer tissues and the expression of Ki-67 in the subcutaneous tumor tissues of nude mice were detected by immunohistochemical staining. All tissue samples were fixed with $30 \%$ formalin at $28^{\circ} \mathrm{C}$ for $60 \mathrm{~min}$ and then embedded with paraffin. The samples were sectioned to a thickness of 5-mm. The sections were incubated at $60^{\circ} \mathrm{C}$ for $1 \mathrm{~h}$, then, placed in xylene I and xylene II for $30 \mathrm{~min}$ in turn. All the slices were dehydrated in a graded alcohol series (100, 95, 90 and 85\%). Slides were placed in $0.01 \mathrm{M}$ potassium citrate solution and microwaved at $90^{\circ} \mathrm{C}$ for $10 \mathrm{~min}$ prior to cooling at room temperature. Slides were then rinsed in PBS 3 times. Endogenous peroxidase activity was blocked with $3 \% \mathrm{H}_{2} \mathrm{O}_{2}$ and goat serum (Haoranbio, Shanghai, China) at $37^{\circ} \mathrm{C}$ for $30 \mathrm{~min}$, then immediately incubated with anti-RIP140 primary antibody 


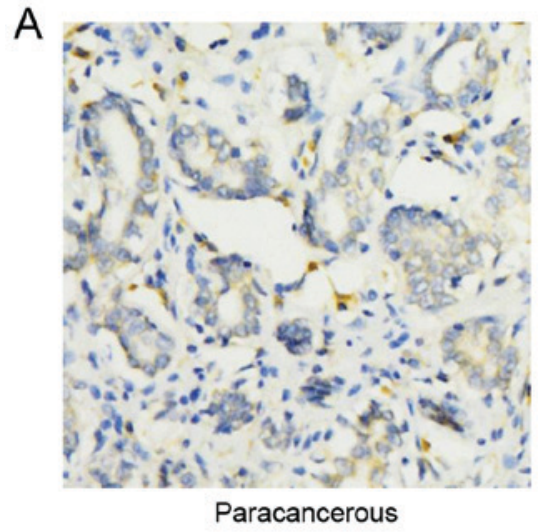

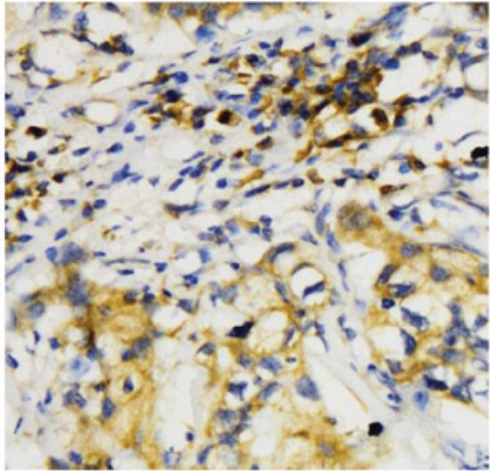

Non-TNBC

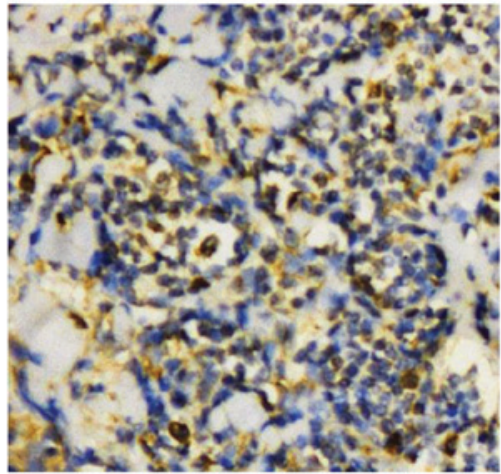

TNBC

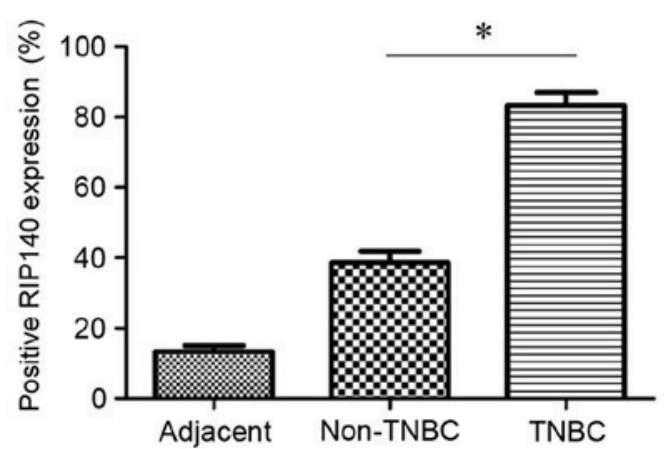

B

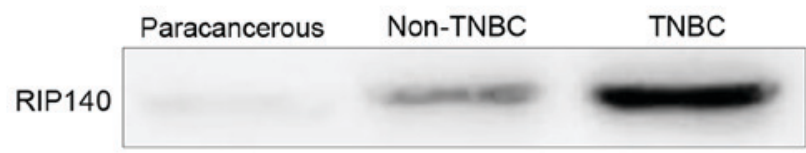

GAPDH

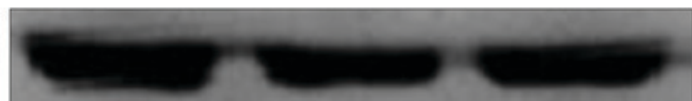

Figure 1. High expression of RIP140 in TNBC tissues. (A) Immunohistochemistry was used to detect the expression of RIP140 in paracancerous, non-TNBC and TNBC breast tissues (magnification, x200). (B) Western blotting was used to detect the protein expression levels of RIP140 in the three tissue types. ${ }^{*} \mathrm{P}<0.05$. RIP140, receptor interacting protein 140; TNBC, triple negative breast cancer.

(dilution, 1:500; catalog no., ab42126; Abcam) or anti-Ki-67 primary antibody (dilution, 1:100; catalog no., sc56319; Santa Cruz Biotechnology, Inc.) at $4^{\circ} \mathrm{C}$ overnight. The next day, the slides were washed with PBS 3 times prior to incubation with corresponding HRP-conjugated mouse anti-goat secondary antibody (dilution, 1:100; catalog no., sc-516246; Santa-Cruz Biotechnology, Inc.) at $37^{\circ} \mathrm{C}$ for $1 \mathrm{~h}$. The slides were then washed three more times in PBS. The slides were stained with DAB (Hengyuan Bio, Shanghai, China) at room temperature for 3-5 min, then rinsed 3 times with tap water, and dyed again prior to sealing using mounting medium (Hengyuan Bio). Images were captured using a Leica DMLA light microscope (Leica Microsystems, Inc., Buffalo Grove, IL, USA). Brown nuclear staining identified RIP140-positive cells. Tissues with $<50 \%$ positive staining were considered to exhibit weakly positive RIP140 expression, while tissues with $>50 \%$ positive staining were considered to exhibit strongly positive RIP140 expression (9).

Statistical analysis. Statistical analyses were performed using SPSS 16.0 software (SPSS, Inc., Chicago, IL, USA). Statistical differences between two groups were determined by Student's t-test. To compare $\geq 3$ groups, one-way analysis of variance followed by Bonferroni's post-hoc test was used when all assumptions (equal variance and normal distribution) were satisfied. All data are expressed as the mean \pm standard deviation. The Kaplan-Meier method and log-rank test were used to perform survival analysis. $\mathrm{P}<0.05$ was considered to indicate a statistically significant difference.

\section{Results}

High expression of RIP140 in TNBC. As depicted in Fig. 1A, weakly positive RIP140 expression was exhibited by paracan-

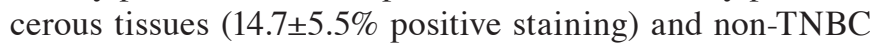
tissues (39.6 $\pm 10.1 \%$ positive staining), whereas TNBC tissues exhibited strongly positive expression in TNBC tissues ( $83.5 \pm 11.4 \%$ positive staining). The expression of RIP140 was significantly increased in TNBC tissues compared with non-TNBC tissues $(\mathrm{P}<0.05)$. To verify these results, western blotting was used to determine the protein expression level of RIP140 in three groups of tissue. As demonstrated in Fig. 1B, the protein expression level of RIP140 in TNBC was increased compared with non-TNBC tissues, and the expression of RIP140 was lowest in paracancerous tissues.

The association of RIP140 expression with clinicopathological features. As demonstrated in Table I, there was a significant difference between the expression of RIP140 in TNBC tissues and non-TNBC tissues $(\mathrm{P}<0.05)$. However, there was no difference in age, tumor size or lymph node metastasis between the two groups $(\mathrm{P}>0.05)$.

The association of RIP140 expression with postoperative prognosis of patients with breast cancer. Patient follow-up data were used to analyze the association of TNBC vs. non-TNBC, and weak vs. high RIP140 expression, with postoperative prognosis of the patients. As depicted in Fig. 2A, the median survival time of patients with TNBC was $34.2 \pm 5.1$ months, whereas the median survival time of patients with non-TNBC 
A

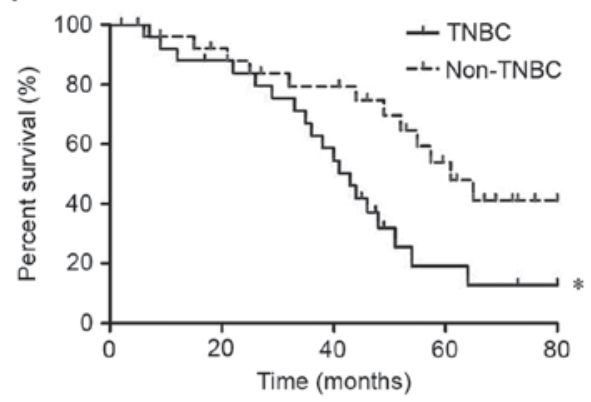

B

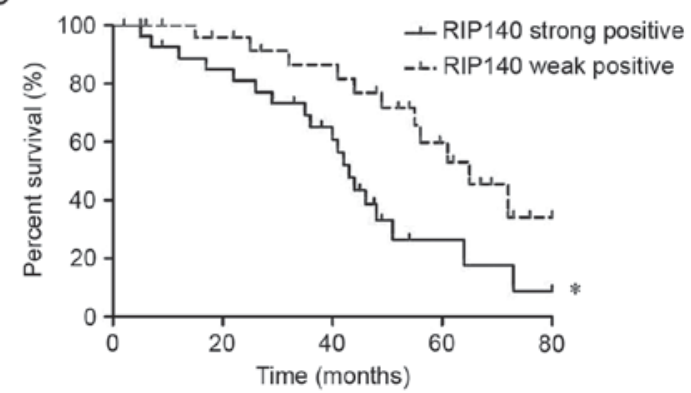

Figure 2. Association of RIP140 expression with postoperative prognosis of patients with TNBC. (A) Postoperative survival curves demonstrating the survival time of patients with TNBC or non-TNBC. (B) Postoperative survival curve analysis demonstrating the survival times of breast cancer patients with weak or strong RIP140 expression. * $\mathrm{P}<0.05$. RIP140, receptor interacting protein 140; TNBC, triple negative breast cancer.

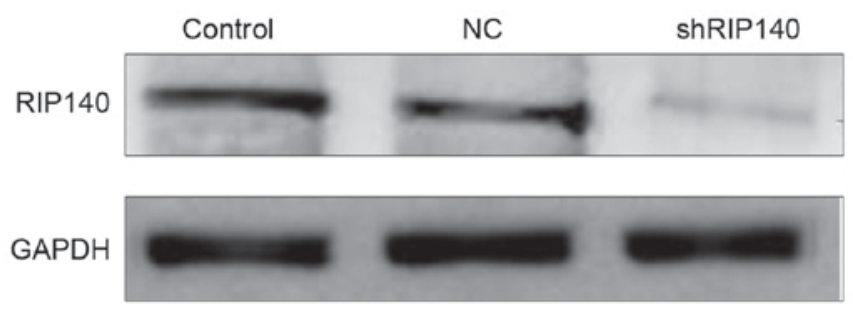

Figure 3. Lentiviral silencing of RIP140 in MDA-MB-231 cells. Western blotting demonstrates the effect of RIP140 silencing on RIP140 protein expression in MDA-MB-231 cells. RIP140, receptor interacting protein 140; NC, negative control; shRIP140, short hairpin RNA targeting RIP140.

was $62.5 \pm 7.4$ months. The prognosis of patients with TNBC was relatively poor compared with that of non-TNBC patients $(\mathrm{P}=0.043)$. As depicted in Fig. 2B, the median survival time was $33.8 \pm 6.3$ months in breast cancer patients exhibiting strong RIP140 expression compared with $63.2 \pm 10.3$ months in patients exhibiting weak RIP140 expression. The overall survival rate of patients exhibiting strong positive expression of RIP140 was low compared with patients exhibiting weakly positive expression of RIP140 $(\mathrm{P}=0.046)$.

The expression of RIP140 in MDA-MB-231 cells significantly decreases following transfection with shRNA-RIP140. After $72 \mathrm{~h}$ of transfection, western blotting was used to analyze the protein expression level of RIP140 in cells of the three groups. As depicted in Fig. 3, the protein expression level of RIP140 in the shRNA-RIP140 group was significantly lower than that in the $\mathrm{NC}$ and control groups $(\mathrm{P}<0.05)$.
A

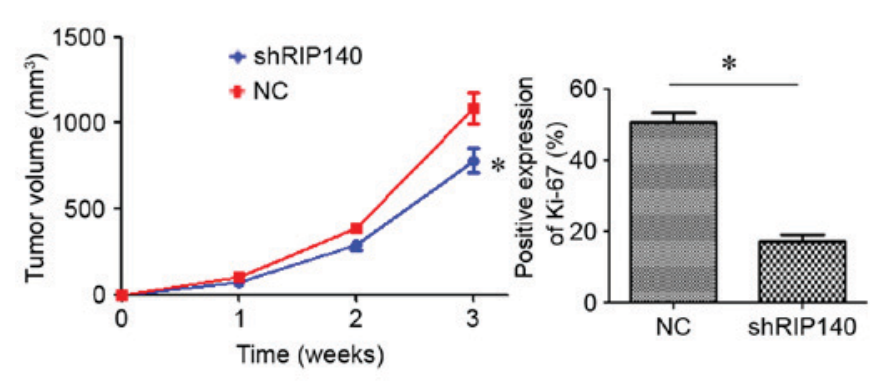

B
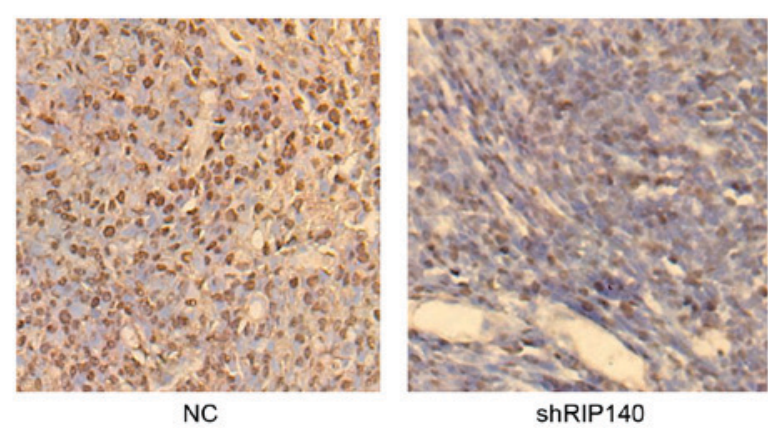

Figure 4. Effects of silencing RIP140 in MDA-MB-231 cells in subcutaneous tumors in BALB/c nude mice. (A) The volumes of subcutaneous tumors in $\mathrm{BALB} / \mathrm{c}$ nude mice at different time points in the shRIP140 and NC groups. (B) Immunohistochemistry was used to detect the expression of Ki-67 in the shRIP140 and NC groups (magnification, x200). ${ }^{*} \mathrm{P}<0.05$. RIP140, receptor interacting protein 140; shRIP140, short hairpin RNA targeting RIP140; $\mathrm{NC}$, negative control.

Downregulation of RIP140 expression in MDA-MB-231 cells inhibits the growth and proliferation of TNBC cells in $B A L B / c$ nude mice. The results of immunohistochemistry and western blotting in clinical breast cancer samples demonstrated that RIP140 was highly expressed in TNBC tissues, and that RIP140 expression was negatively associated with postoperative prognosis, indicating that high expression of RIP140 accelerated the development of breast cancer. To investigate whether silencing the expression of RIP140 inhibits the occurrence and development of TNBC, RIP140 was downregulated in MDA-MB-231 cells using a shRNA-RIP140 lentivirus, and used to generate subcutaneous tumors in mice. The tumor volume of the shRNA-RIP140 group was significantly lower than that of NC group $(\mathrm{P}<0.05$; Fig. 4A). The expression intensity of $\mathrm{Ki}-67$ in the shRNA-RIP140 group was lower than that in the $\mathrm{NC}$ group $(\mathrm{P}<0.05$; Fig. 4B). These results indicated that downregulation of RIP140 in TNBC MDA-MB-231 cells may inhibit the growth of TNBC.

\section{Discussion}

Breast cancer is among the most common types of malignant tumor worldwide, and presents a serious threat to the physical and mental health of women $(1,2)$. In 2014, the number of new cases of breast cancer in China accounted for $12.2 \%$ of the new cases worldwide, and the number of mortalities due to breast cancer accounted for $\sim 9.6 \%$ of the number mortalities due to breast cancer worldwide (10). At present, the major treatment modalities for breast cancer include surgical resection, 
chemotherapy, radiotherapy and biological therapy $(11,12)$. Patients with non-TNBC have a better prognosis when diagnosed and treated early (13); however, TNBC is characterized by an early age of onset, high degree of malignancy, high recurrence rate, early metastasis and poor prognosis (14). Previous research has demonstrated that patients with TNBC have a median survival time of 4.2 years between the date of surgery and the date of mortality, which is significantly lower than the survival time of non-TNBC patients (6 years) $(14,15)$. Thus, the identification of an effective therapeutic target for TNBC is urgently required.

RIP140 serves notable roles in metabolism, the inflammatory response and tumor development (4-6). Recent studies have demonstrated that RIP140 expression, and the associated pathological effects, vary between types of tumor. Zhang et al (16) observed that RIP140 was expressed at low expression in liver cancer, and that downregulation of RIP140 expression in hepatocellular cells promoted the development of liver cancer. Upregulation of RIP140 expression in hepatocellular carcinoma cells inhibited the growth and proliferation of liver cancer. RIP140 was hypothesized to negatively regulate the $\beta$-catenin/T-cell factor signaling pathway, thus inhibiting the growth of hepatocellular carcinoma cells. Lapierre et al (17) revealed that expression of RIP140 in colon carcinoma was low, and that overexpression of RIP140 in colon cancer cells promoted the expression of adenomatous polyposis coli, which inhibited the growth and metastasis of colon cancer by inhibiting the Wnt/APC/ $\beta$-catenin signaling pathway. However, Aziz et al (8) demonstrated that RIP140 was highly expressed in breast cancer tissues, and that inhibition of RIP140 expression in breast cancer cells inhibited growth, proliferation and invasion in vivo and in vitro.

In the present study, RIP140 expression was investigated in TNBC tissues and non-TNBC tissues. The association between RIP140 expression and the postoperative prognosis of patients with breast cancer was also studied. The expression of RIP140 in MDA-MB-231 cells was silenced using a shRNA, and the cells were subcutaneously injected into BALB/c nude mice to establish an in vivo TNBC model. The results demonstrated that RIP140 expression in TNBC tissue was significantly higher than that in non-TNBC tissue, and that RIP140 expression was higher in the two types of breast cancer tissue than in paracancerous tissue. Silencing the expression of RIP140 in MDA-MB-231 cells inhibited the growth and proliferation of subcutaneous tumors in BALB/c nude mice. Furthermore, the overall survival rate of patients exhibiting strong RIP140 expression was lower than that of patients exhibiting weak RIP140 expression.

In summary, data from the present and previous studies indicated that, different types of tumor tissue exhibited varying levels of expression of RIP140, with low expression in liver cancer and colon cancer, and high expression in breast cancer tissues. The abnormal expression of RIP140 in different tumor tissues was associated with the risk of occurrence and development of tumors $(5,8)$. The present study confirmed that the abnormal expression of RIP140 is associated with the malignant biological behavior and prognosis of TNBC, serving a notable role in the growth and proliferation of TNBC cells. The present study also indicated that RIP140 represents a novel therapeutic target for TNBC.

\section{References}

1. Warner E: Clinical practice, breast-cancer screening. N Eng J Med 365: 1025-1032, 2011.

2. Zeichner SB, Terawaki H and Gogineni K: A review of systemic treatment in metastatic triple-negative breast cancer. Breast Cancer (Auckl) 10: 25-36, 2016.

3. Collignon J, Lousberg L, Schroeder H and Jerusalem G: Triple-negative breast cancer: Treatment challenges and solutions. Breast Cancer (Dove Med Press) 8: 93-107, 2016.

4. Rosell M, Jones MC and Parker MG: Role of nuclear receptor corepressor RIP140 in metabolic syndrome. Biochim Biophys Acta 1812: 919-928, 2011.

5. Docquier A, Harmand PO, Fritsch S, Chanrion M, Darbon JM and Cavaillès V: The transcriptional coregulator RIP140 represses E2F1 activity and discriminates breast cancer subtypes. Clin Cancer Res 16: 2959-2970, 2010.

6. Ho PC, Tsui YC, Feng X, Greaves DR and Wei LN: NF-kB mediated degradation of the co-activator RIP140 regulates inflammatory response and contributes to endotoxin tolerance. Nat Immunol 13: 379-386, 2012.

7. Herbst EA, Bonen A and Holloway GP: Changes in nuclear receptor corepressor RIP140 do not influence mitochondrial content in the cortex. Appl Physiol Nutr Metab 40: 1086-1088, 2015.

8. Aziz MH, Chen X, Zhang Q, DeFrain C, Osland J, Luo Y, Shi X and Yuan R: Suppressing NRIP1 inhibits growth of breast cancer cells in vitro and in vivo. Oncotarget 6: 39714-39724, 2015.

9. Fu F, Xiao XI, Zhang T, Zou Q, Chen Z, Pei L, Su J and Yi WJ: Expression of receptor protein tyrosine phosphatase $\zeta$ is a risk factor for triple negative breast cancer relapse. Biomed Rep 4: 167-172, 2016.

10. Fan L, Strasser-Weippl K, Li JJ, St Louis J, Finkelstein DM, Yu KD, Chen WQ, Shao ZM and Goss PE: Breast cancer in China. Lancet Oncol 15: e279-e289, 2014.

11. Piroth MD, Petz D, Pinkawa M, Holy R and Eble MJ: Usefulness of a thermoplastic breast bra for breast cancer radiotherapy: A prospective analysis. Strahlenther Onkol 192: 609-616, 2016.

12. Moloney N, Sung JM, Kilbreath S and Dylke E: Prevalence and risk factors associated with pain 21 months following surgery for breast cancer. Support Care Cancer 24: 4533-4539, 2016.

13. Mangolini A, Ferracin M, Zanzi MV, Saccenti E, Ebnaof SO, Poma VV, Sanz JM, Passaro A, Pedriali M, Frassoldati A, et al: Diagnostic and prognostic microRNAs in the serum of breast cancer patients measured by droplet digital PCR. Biomark Res 3: $12,2015$.

14. Dent R, Trudeau M, Pritchard KI, Hanna WM, Kahn HK, Sawka CA, Lickley LA, Rawlinson E, Sun P and Narod SA: Triple-negative breast cancer: Clinical features and patterns of recurrence. Clin Cancer Res 13: 4429-4434, 2007.

15. De P, Carlson JH, Wu H, Marcus A, Leyland-Jones B and Dey N: Wnt-beta-catenin pathway signals metastasis-associated tumor cell phenotypes in triple negative breast cancers. Oncotarget 7 : 43124-43149, 2016.

16. Zhang D, Wang Y, Dai Y, Wang J, Suo T, Pan H, Liu H, Shen S and Liu H: Downregulation of RIP140 in hepatocellular carcinoma promoted the growth and migration of the cancer cells. Tumour Biol 36: 2077-2085, 2015.

17. Lapierre M, Bonnet S, Bascoul-Mollevi C, Ait-Arsa I, Jalaguier S, Del Rio M, Plateroti M, Roepman P, Ychou M, Pannequin J, et al: RIP140 increases APC expression and controls intestinal homeostasis and tumorigenesis. J Clin Invest 124: 1899-1913, 2014. 